\title{
Stress-deformed state in the plane domain boundary angle cutout zone
}

\author{
Ludmila Frishter ${ }^{1, *}$ \\ ${ }^{1}$ Moscow State University of Civil Engineering, Yaroslavskoe shosse, 26, Moscow, 129337, Russia
}

\begin{abstract}
Studying the stress-deformed state of structures with complexshaped boundary is an urgent task for designing and developing numerical and analytical methods of constructions, buildings and structures research. Stress concentration due to geometrically nonlinear shape of the boundaries - cutouts, notches, causes stress, deformation zones with significant magnitudes and gradients. Theoretical analysis of the boundary angle cutout domain stress-deformed state (SDS) under rupturing forced deformations comes down to studying singular solutions of elasticity theory homogeneous problem with power-type specifics. Novelty of the studies given herein is that the SDS in the vicinity of an irregular boundary point - the domain boundary angle cutout vertex, is characterized by stress limit values similar to stress intensity coefficients when applying force criteria in fracture mechanics. Asymptotics of elastic problem solution in the random opening boundary angle cutout domain is written using the stress limit values. Obtained asymptotic expression for elasticity theory plane problem solution allows to analyze the SDS of the domain boundary angle cutout zone as a function of the domain cutout opening angle, mechanical characteristics and eigenvalues of elasticity boundary-value problem in case of stresses homogeneous boundary conditions.
\end{abstract}

\section{Introduction}

Stress-deformed state (SDS) of a composite domain in the boundary angle cutout zones under forced deformations with a finite rupture of forced deformations along the contact line (surface) of the domain elements entering the boundary cutout vertex is considered. This paper is aimed at determining the SDS in the domain boundary angle point, to which forced deformation rupture enters, using the limit stress values called stress intensity coefficients in a manner similar to the force criteria in fracture mechanics.

Elasticity theory problem in the vicinity of an irregular point on the domain boundary special line comes down to consideration of two plane problems [1-5]: plane deformation and anti-plane deformation or transverse shear.

The SDS specifics conditioned by the domain boundary shape geometry - cutout, notch, is determined by the singularity of solution, the order of which depends on an elasticity theory homogeneous boundary-value problem eigenvalues [4, 6-11]. The eigenvalues of a

\footnotetext{
* Corresponding author: 1frishter@mail.ru
} 
homogeneous boundary-value problem depend on the boundary shape, type of homogeneous boundary conditions, mechanical characteristics of the domain material and have a set of values [12-14]. Let's consider solution of a plane deformation elastic problem in the vicinity of the plane domain boundary angle cutout vertex in more detail.

\section{Materials and Methods}

\subsection{Problem statement and solution}

To obtain an asymptotic solution in the vicinity of the boundary irregular point - the angle cutout vertex, we consider the solution of a homogeneous boundary-value problem for wedge. A plane wedge-shaped domain with the opening $2 \alpha \in(\pi, 2 \pi)$ consists of two symmetrical domains: $\Omega_{1}, \theta \in[0, \alpha]$ and $\Omega_{2}, \theta \in[-\alpha, 0]$, in one of which there are forced temperature deformations $\varepsilon_{i j}=\alpha T \delta_{i j}$. A deformation discontinuity (jump) $\Delta \varepsilon_{i j}=\alpha T \delta_{i j}$ appears along the domains interface $L=\Omega_{1} \cap \Omega_{2}$ entering the domain boundary angle cutout vertex. Stresses boundary conditions are homogeneous. Concentrated forces are not considered.

Let's introduce a polar coordinate system with the polar pole $O(0.0)$ at the boundary angle cutout vertex and polar axis along the wedge symmetry axis. Solution of the homogeneous elastic problem in displacements for a wedge-shaped infinite domain in the vicinity of an arbitrary opening boundary angle point is written $[4,6,8]$ as follows:

$$
u_{r}=r^{\lambda} g(\theta), u_{\theta}=r^{\lambda} f(\theta),
$$

where $\lambda$ is an unknown parameter, $f(\theta), g(\theta)$ are unknown functions of the angle $\theta$ to be determined. By substituting (1) in the Lame equations, we obtain the SDS, which depend on four arbitrary constants $A, B, C, D$ to be determined:

$$
\begin{gathered}
\mathrm{r}^{-\lambda} \mathrm{u}_{r}=A \cos [(1+\lambda) \theta]+B \sin [(1+\lambda) \theta]+C \cos [(1-\lambda) \theta]+D \sin [(1-\lambda) \theta], \\
\mathrm{r}^{-\lambda} \mathrm{u}_{\theta}=B \cos [(1+\lambda) \theta]-A \sin [(1+\lambda) \theta]+v_{2} D \cos [(1-\lambda) \theta]-v_{2} C \sin [(1-\lambda) \theta], \\
\mu^{-1} \mathrm{r}^{1-\lambda} \sigma_{\theta}=-2 \lambda A \cos [(1+\lambda) \theta]-2 \lambda B \sin [(1+\lambda) \theta]- \\
\quad-(1+\lambda)\left(1-v_{2}\right) C \cos [(1-\lambda) \theta]-(1+\lambda)\left(1-v_{2}\right) D \sin [(1-\lambda) \theta] \\
\mu^{-1} \mathrm{r}^{1-\lambda} \tau_{r \theta}=-2 \lambda A \sin [(1+\lambda) \theta]+2 \lambda B \cos [(1+\lambda) \theta]- \\
\quad-(1-\lambda)\left(1-v_{2}\right) C \sin [(1-\lambda) \theta]+(1-\lambda)\left(1-v_{2}\right) D \cos [(1-\lambda) \theta] \\
\mu^{-1} \mathrm{r}^{\lambda-1} \sigma_{r}=2 \lambda\{(A \cos [(1+\lambda) \theta]+B \sin [(1+\lambda) \theta]+ \\
\left.+\frac{3-\lambda}{k-\lambda} C \cos [(1-\lambda) \theta]+\frac{3-\lambda}{k-\lambda} \mathrm{D} \sin [(1-\lambda) \theta]\right\}
\end{gathered}
$$

where $\mu=\frac{E}{2(1+v)}, E, \quad v$ is an elastic modulus and domain material Poisson's ratio, respectively; $\quad v_{2}=\frac{3+\lambda-4 v}{3-\lambda-4 v}, \quad 1-v_{2}=\frac{-2 \lambda}{k-\lambda} \quad k=3-4 v, \quad \lambda$ are the homogeneous boundary-value problem eigenvalues. 
By satisfying the homogeneous boundary conditions: $\sigma_{\theta}=\tau_{r \theta}=0$ with $\theta= \pm \alpha$, we obtain two homogeneous systems of linear equations:

$$
\begin{aligned}
& \left\{\begin{array}{l}
2 \lambda A \cos [(1+\lambda) \alpha]+(1+\lambda)\left(1-v_{2}\right) C \cos [(1-\lambda) \alpha]=0, \\
2 \lambda A \sin [(1+\lambda) \alpha]+(1-\lambda)\left(1-v_{2}\right) C \sin [(1-\lambda) \alpha]=0,
\end{array}\right. \\
& \left\{\begin{array}{l}
2 \lambda B \sin [(1+\lambda) \alpha]+(1+\lambda)\left(1-v_{2}\right) D \sin [(1-\lambda) \alpha]=0, \\
2 \lambda B \cos [(1+\lambda) \alpha]+(1-\lambda)\left(1-v_{2}\right) D \cos [(1-\lambda) \alpha]=0,
\end{array}\right.
\end{aligned}
$$

For the homogeneous boundary-value problem to have non-zero solutions, it is necessary and sufficient that the determinants of each system (3a) and (3b) are equal to zero. The determinant of the first system ( $3 a$ ) is equal to:

$$
\lambda \sin 2 \alpha+\sin 2 \lambda \alpha=0 \quad \text { or } \quad \sin 2 \lambda \alpha=-\lambda \sin 2 \alpha .
$$

Only the components $\mathrm{A}$ and $\mathrm{C}(\mathrm{B}, \mathrm{D}=0)$ written for the characteristic equation (5) $\lambda$ roots are retained (kept) in the SDS of the form (2). Let's designate the equation root system (4) with $\lambda_{i}^{-}$.

The determinant of the second linear equations system (3b):

$$
-\lambda \sin 2 \alpha+\sin 2 \lambda \alpha=0 \quad \text { or } \quad \sin 2 \lambda \alpha=\lambda \sin 2 \alpha
$$

Only the components $\mathrm{B}$ and $\mathrm{D}(\mathrm{A}, \mathrm{C}=0)$ written for the characteristic equation (5) $\lambda$ roots are retained (kept) in the SDS of the form (2). Let's designate the equation root system (5) with $\lambda_{i}^{+}$.

The characteristic equations (4), (5) have an infinite set of eigenvalues $\lambda_{i}^{+}, \lambda_{i}^{-}$. The values $\lambda<0$ result in unlimited movements at the angle cutout vertex when there are no loads on the domain cutout edge. The values $\lambda_{i}>1$ result in unlimited stresses at infinity. According to the problem physical meaning, the values $\lambda_{i} \in[0,1]$ are taken. Among the set of roots (4), (5) $\lambda^{+}=\min \operatorname{Re} \lambda_{i}^{+}, \lambda^{-}=\min \operatorname{Re} \lambda_{i}^{-}$is chosen to construct the asymptotic solution (2), since the subsequent higher values $\lambda_{i}$ result in unlimited increase in deformation energy.

Taking into account the boundary conditions (3a), we obtain ratios for the coefficients A and C:

$$
A=\frac{\left(1-\lambda^{-}\right) \sin \left[\left(1-\lambda^{-}\right) \alpha\right]}{\left(k-\lambda^{-}\right) \sin \left[\left(1+\lambda^{-}\right) \alpha\right]} C .
$$

Taking into account the boundary conditions (3b), we obtain ratios for the coefficients $B$ and D:

$$
B=\frac{\left(1+\lambda^{+}\right) \sin \left[\left(1-\lambda^{+}\right) \alpha\right]}{\left(k-\lambda^{+}\right) \sin \left[\left(1+\lambda^{+}\right) \alpha\right]} D .
$$

Let's designate the stresses limit values in the vicinity of the domain boundary irregular point as follows:

$$
\begin{aligned}
& \mathrm{K}_{I}=\lim _{r \rightarrow 0} r^{1-\lambda^{-}} \sigma_{\theta, \theta=0} . \\
& \mathrm{K}_{I I}=\lim _{r \rightarrow 0} r^{1-\lambda^{+}} \tau_{r \theta, \theta=0} .
\end{aligned}
$$

Taking into account the designations (8), (9) and ratios (6), (7), the coefficients A and B, C and $\mathrm{D}$ are expressed in terms of the stress limit values as follows: 


$$
\begin{aligned}
& A=\frac{\left(1-\lambda^{-}\right) \sin \left[\left(1-\lambda^{-}\right) \alpha\right]}{2 \lambda^{-} \mu\left[\left(\lambda^{-}-1\right) \sin \left[\left(1-\lambda^{-}\right) \alpha\right]+\left(\lambda^{-}+1\right) \sin [(1+\lambda) \alpha]\right]} K_{I}, \\
& C=\frac{\left(\mathrm{k}-\lambda^{-}\right) \sin \left[\left(1+\lambda^{-}\right) \alpha\right]}{2 \lambda^{-} \mu\left[\left(\lambda^{-}-1\right) \sin \left[\left(1-\lambda^{-}\right) \alpha\right]+\left(\lambda^{-}+1\right) \sin \left[\left(1+\lambda^{-}\right) \alpha\right]\right]} K_{I}, \\
& \mathrm{~B}=\frac{\left(1+\lambda^{+}\right) \sin \left[\left(1-\lambda^{+}\right) \alpha\right]}{2 \lambda^{+} \mu\left[\left(\lambda^{+}+1\right) \sin \left[\left(1-\lambda^{+}\right) \alpha\right]-\left(1-\lambda^{+}\right) \sin \left[\left(1+\lambda^{+}\right) \alpha\right]\right]} K_{I I}, \\
& D=\frac{\left(\mathrm{k}-\lambda^{+}\right) \sin \left[\left(1+\lambda^{+}\right) \alpha\right]}{2 \lambda^{+} \mu\left[\left(\lambda^{+}+1\right) \sin \left[\left(1-\lambda^{+}\right) \alpha\right]-\left(1-\lambda^{+}\right) \sin \left[\left(1+\lambda^{+}\right) \alpha\right]\right]} K_{I I} .
\end{aligned}
$$

\subsection{Stress-deformed state in the vicinity of the plane domain boundary angle cutout vertex}

Solution of the homogeneous boundary-value problem (2) considering the coefficients (10) in the vicinity of the domain boundary angle cutout vertex is written as follows:

$$
\begin{gathered}
\mathrm{u}_{r}=\mathrm{r}^{\lambda^{-}}\left\{\mathrm{Acos}\left[\left(1+\lambda^{-}\right) \theta\right]+C \cos \left[\left(1-\lambda^{-}\right) \theta\right]\right\}+ \\
+\mathrm{r}^{\lambda^{+}}\left\{B \sin \left[\left(1+\lambda^{+}\right) \theta\right]+D \sin \left[\left(1-\lambda^{+}\right) \theta\right]\right. \\
\mathrm{u}_{\theta}=\mathrm{r}^{\lambda^{-}}\left\{-A \sin \left[\left(1+\lambda^{-}\right) \theta\right]-v_{2}^{-} C \sin \left[\left(1-\lambda^{-}\right) \theta\right]\right\}+ \\
+\mathrm{r}^{\lambda^{+}}\left\{B \cos \left[\left(1+\lambda^{+}\right) \theta\right]+v_{2}^{+} D \cos \left[\left(1-\lambda^{+}\right) \theta\right]\right\} \\
\sigma_{\theta}=\mu \mathrm{r}^{\lambda^{-}-1}\left\{-2 \lambda^{-} A \cos \left[\left(1+\lambda^{-}\right) \theta\right]-\left(1+\lambda^{-}\right)\left(1-v_{2}^{-}\right) C \cos \left[\left(1-\lambda^{-}\right) \theta\right]\right\}+ \\
+\mu \mathrm{r}^{\lambda^{+}-1}\left\{-2 \lambda^{+} B \sin \left[\left(1+\lambda^{+}\right) \theta\right]-\left(1+\lambda^{+}\right)\left(1-v_{2}^{+}\right) D \sin \left[\left(1-\lambda^{+}\right) \theta\right]\right\} \\
\tau_{r \theta}=\mu \mathrm{r}^{\lambda^{-}-1}\left\{-2 \lambda^{-} A \sin \left[\left(1+\lambda^{-}\right) \theta\right]-\left(1-\lambda^{-}\right)\left(1-v_{2}^{-}\right) C \sin \left[\left(1-\lambda^{-}\right) \theta\right]+\right. \\
+\mu \mathrm{r}^{\lambda^{+}-1}\left\{2 \lambda^{+} B \cos \left[\left(1+\lambda^{+}\right) \theta\right]+\left(1-\lambda^{+}\right)\left(1-v_{2}^{+}\right) D \cos \left[\left(1-\lambda^{+}\right) \theta\right],\right. \\
\sigma_{r}=2 \mu \lambda \mathrm{r}^{\lambda^{-}-1}\left\{\left(A \cos \left[\left(1+\lambda^{-}\right) \theta\right]+\frac{3-\lambda^{-}}{k-\lambda^{-}} C \cos \left[\left(1-\lambda^{-}\right) \theta\right]\right\}+\right. \\
+2 \mu \lambda \mathrm{r}^{\lambda^{+}-1}\left\{B \sin \left[\left(1+\lambda^{+}\right) \theta\right]+\frac{3-\lambda^{+}}{k-\lambda^{+}} \mathrm{Dsin}\left[\left(1-\lambda^{+}\right) \theta\right]\right\}
\end{gathered}
$$

where the coefficients A, B, C, D satisfy the ratios (10).

Taking into account the set of eigenvalues $\lambda_{i}$ of the equation solutions (4) and (5), solution of the elastic problem in the plane domain boundary angle cutout zone is represented as follows: 


$$
\begin{gathered}
\mathrm{u}_{r}=\sum_{i, \lambda_{i}^{-}} \mathrm{r}^{\lambda_{i}^{-}}\left\{\mathrm{A}_{i} \cos \left[\left(1+\lambda_{i}^{-}\right) \theta\right]+C_{i} \cos \left[\left(1-\lambda_{i}^{-}\right) \theta\right]\right\}+ \\
+\sum_{i, \lambda_{i}^{+}} \mathrm{r}_{i}^{+}\left\{B_{i} \sin \left[\left(1+\lambda_{i}^{+}\right) \theta\right]+D_{i} \sin \left[\left(1-\lambda_{i}^{+}\right) \theta\right]+\mathrm{u}_{r}^{s}\right. \\
\mathrm{u}_{\theta}=\sum_{i, \lambda_{i}^{-}} \mathrm{r}^{\lambda_{i}^{-}}\left\{-A_{i} \sin \left[\left(1+\lambda_{i}^{-}\right) \theta\right]-v_{2}^{-} C_{i} \sin \left[\left(1-\lambda_{i}^{-}\right) \theta\right]\right\}+ \\
+\sum_{i, \lambda_{i}^{+}} \mathrm{r}_{i}^{\lambda_{i}^{+}}\left\{B_{i} \cos \left[\left(1+\lambda_{i}^{+}\right) \theta\right]+v_{2}^{+} D_{i} \cos \left[\left(1-\lambda_{i}^{+}\right) \theta\right]\right\}+\mathrm{u}_{\theta}^{s} \\
\sigma_{\theta}=\mu \sum_{i, \lambda_{i}^{-}} \mathrm{r}^{\lambda^{-}-1}\left\{-2 \lambda_{i}^{-} A_{i} \cos \left[\left(1+\lambda_{i}^{-}\right) \theta\right]-\left(1+\lambda_{i}^{-}\right)\left(1-v_{2}^{-}\right) C_{i} \cos \left[\left(1-\lambda_{i}^{-}\right) \theta\right]\right\}+ \\
+\mu \sum_{i, \lambda_{i}^{+}} \mathrm{r}^{\lambda^{+}-1}\left\{-2 \lambda_{i}^{+} B_{i} \sin \left[\left(1+\lambda_{i}^{+}\right) \theta\right]-\left(1+\lambda_{i}^{+}\right)\left(1-v_{2}^{+}\right) D \sin \left[\left(1-\lambda_{i}^{+}\right) \theta\right]\right\}+\sigma_{\theta}^{s} \\
\tau_{r \theta}=\mu \sum_{i, \lambda_{i}^{-}} \mathrm{r}^{\lambda^{-}-1}\left\{-2 \lambda_{i}^{-} A_{i} \sin \left[\left(1+\lambda_{i}^{-}\right) \theta\right]-\left(1-\lambda_{i}^{-}\right)\left(1-v_{2}^{-}\right) C_{i} \sin \left[\left(1-\lambda_{i}^{-}\right) \theta\right]\right. \\
+\mu \sum_{i, \lambda_{i}^{+}} \mathrm{r}_{i}^{\lambda_{i}^{+}-1}\left\{2 \lambda_{i}^{+} B_{i} \cos \left[\left(1+\lambda_{i}^{+}\right) \theta\right]+\left(1-\lambda_{i}^{+}\right)\left(1-v_{2}^{+}\right) D_{i} \cos \left[\left(1-\lambda_{i}^{+}\right) \theta\right]+\tau_{r \theta}^{s}\right. \\
\sigma_{r}=2 \mu \sum_{i, \lambda_{i}^{-}} \lambda_{i}^{-} \mathrm{r}^{\lambda_{i}^{-}-1}\left\{\left(A_{i} \cos \left[\left(1+\lambda_{i}^{-}\right) \theta\right]+\frac{3-\lambda_{i}^{-}}{k-\lambda_{i}^{-}} C_{i} \cos \left[\left(1-\lambda_{i}^{-}\right) \theta\right]\right\}+\right. \\
+2 \mu \sum_{i, \lambda_{i}^{+}} \lambda_{i}^{+} \mathrm{r}_{i}^{\lambda_{i}^{+}-1}\left\{B_{i} \sin \left[\left(1+\lambda_{i}^{+}\right) \theta\right]+\frac{3-\lambda_{i}^{+}}{k-\lambda_{i}^{+}} \mathrm{D}_{i} \sin \left[\left(1-\lambda_{i}^{+}\right) \theta\right]\right\}+\sigma_{r}^{s}
\end{gathered}
$$

where $u_{i}^{S}, \sigma_{i j}^{S}$ are the displacements and stresses caused by general displacement and stresses field [12-16] or given forced temperature deformations discontinued along the wedge symmetry axis $\theta=0$ of the following form: $\Delta \varepsilon_{i j}=\alpha T \delta_{i j}$.

According to (10), the coefficients A, B, C, D are expressed in terms of the stress limit values $K_{I}, \mathrm{~K}_{I I}$, therefore stress-deformed state in the vicinity of the domain boundary irregular point depends on the parameters $\mathrm{K}_{I}, \mathrm{~K}_{I I}$ and is written as follows:

$$
\begin{aligned}
u_{i}= & \mu^{-1} \mathrm{~K}_{I} r^{\lambda^{-}} f_{i \lambda^{-}}(\theta)+\mu^{-1} \mathrm{~K}_{I I} r^{\lambda^{+}} f_{i, \lambda^{+}}(\theta)+ \\
& +\sum_{i, \lambda_{i}^{ \pm}}\left[r^{\lambda_{i}^{-}} C_{i} f_{i, \lambda_{i}^{-}}(\theta)+r^{\lambda_{i}^{+}} C_{i} f_{i, \lambda_{i}^{+}}(\theta)\right]+u_{i}^{s}, \\
\sigma_{i j} & =\mathrm{K}_{I} r^{\lambda^{-}} f_{i j, \lambda^{-}}(\theta)+\mathrm{K}_{I I} r^{\lambda^{+}} f_{i j, \lambda^{+}}(\theta)+ \\
& +\mu \sum_{i, j, \lambda_{i}^{+}}\left[r^{\lambda_{i}^{-}} C_{i} f_{i j, \lambda_{i}^{-}}(\theta)+r^{\lambda_{i}^{+}} C_{i} f_{i j, \lambda_{i}^{+}}(\theta)\right]+\sigma_{i j}^{s},
\end{aligned}
$$


where $\lambda^{-}=\min \operatorname{Re} \lambda_{i}^{-}, \lambda^{+}=\min \operatorname{Re} \lambda_{i}^{+}$are the minimum values of the characteristic equations (4), (5) complex roots real part, respectively; $f_{i, \lambda^{ \pm}}(\theta), f_{i j, \lambda^{ \pm}}(\theta)$ are functions of the angle $\theta \in[-\alpha, \alpha], u_{i}=\left(u_{r}, u_{\theta}\right)$ are displacements, $\sigma_{i j}=\left(\sigma_{\theta}, \tau_{r \theta}, \sigma_{r}\right)$ are stresses for which the principal expansion term, written through intensity coefficients $\mathrm{K}_{I}, \mathrm{~K}_{I I}$, is extracted; $u_{i}^{S}, \sigma_{i j}^{s}$ are displacements and stresses caused by specified forced temperature deformations.

Taking into account the SDS of the form (13), the principal term of the problem solution asymptotics in the vicinity of the domain boundary irregular point is as follows:

$$
\begin{array}{r}
u_{i}=\mu^{-1} \mathrm{~K}_{I} r^{\lambda^{-}} f_{i \lambda^{-}}(\theta)+\mu^{-1} \mathrm{~K}_{I I} r^{\lambda^{+}} f_{i, \lambda^{+}}(\theta)+O\left(r^{\lambda}\right), \\
\sigma_{i j}=\mathrm{K}_{I} r^{-1+\lambda^{-}} f_{i j, \lambda^{-}}(\theta)+\mathrm{K}_{I I} r^{-1+\lambda^{+}} f_{i j, \lambda^{+}}(\theta)+o\left(r^{-1+\lambda}\right),
\end{array}
$$

where $\lambda^{-}=\min \operatorname{Re} \lambda_{i}^{-}, \lambda^{+}=\min \operatorname{Re} \lambda_{i}^{+}, \lambda=\min \left(\lambda^{-}, \lambda^{+}\right)$. In the vicinity of the domain boundary irregular point $\mathrm{O}(0.0)$ singularity order for stresses is equal to $(\lambda-1), \lambda \in[0.5 ; 1], \lim _{r \rightarrow 0} r^{\lambda-1}=+\infty$, and the mean value of the stresses $(14 \mathrm{~b})$ in the small vicinity of this point is finite. This follows from convergence of the improper integral of the following form:

$$
\iint_{\Omega_{\varepsilon}} \mathrm{K}_{I} r^{-1+\lambda} f_{i j, \lambda}(\theta)=K_{I} \lim _{n \rightarrow \infty} \iint_{C_{\varepsilon}} f_{i U_{n}}(\theta) \frac{r d r d \theta}{r^{1-\lambda}}=K_{I} \lim _{n \rightarrow \infty} \int_{\rho_{n}}^{1} \int_{-\alpha}^{\alpha} f_{i j, \lambda}(\theta) r^{\lambda} d r d \theta .
$$

where $\left\{\rho_{n}\right\}$ is the sequence of vicinities centered at the beginning of the polar coordinate system (excluding the boundary cutout vertex) in the form of central sectors which radii $\rho_{n}, \lim _{n \rightarrow \infty} \rho_{n}=0$. For function of the angle $f_{i j, \lambda}(\theta)=1$, the improper integral value:

$$
\left.\frac{2 \alpha}{1+\lambda} \lim _{n \rightarrow \infty} \rho^{\lambda+1}\right|_{\rho_{n}} ^{1}=\frac{2 \alpha}{1+\lambda} K_{I}\left(K_{I I}\right)
$$

Taking into account general stress field from the given forced deformations and homogeneous problem solution asymptotics (14), the SDS in the vicinity of the domain boundary irregular point is as follows:

$$
\begin{array}{r}
u_{i} \cong \mu^{-1} \mathrm{~K}_{I} r^{\lambda^{-}} f_{i, \lambda^{-}}(\theta)+\mu^{-1} \mathrm{~K}_{I I} r^{\lambda^{+}} f_{i, \lambda^{+}}(\theta)+u_{i}^{s}, \\
\sigma_{i j} \cong \mathrm{K}_{I} r^{-1+\lambda^{-}} f_{i j, \lambda^{-}}(\theta)+\mathrm{K}_{I I} r^{-1+\lambda^{+}} f_{i j, \lambda^{+}}(\theta)+\sigma_{i j}^{s},
\end{array}
$$

where $\lambda^{-}=\min \operatorname{Re} \lambda_{i}^{-}, \lambda^{+}=\min \operatorname{Re} \lambda_{i}^{+}$, coefficients $\mathrm{K}_{I}, \mathrm{~K}_{I I}$ are the stress limit values of the form (8), (9).

\section{Conclusions}

A solution of the elasticity theory boundary-value problem for a boundary angle cutout domain in the form (12), taking into account differences in the minimal values of the characteristic equation root real parts (4), (5), is obtained. 
The SDS in the plane domain boundary angle point which is entered by forced deformations rupture in the form (13) using the stress values (8), (9), called stress intensity coefficients similar to the force criteria in fracture mechanics, is obtained.

The principal term of elasticity theory boundary-value problem solution asymptotics in the vicinity of a domain boundary irregular point with an arbitrary opening of the boundary entering angle cutout in the form (14) is given. The obtained solution of the elastic problem (14) allows to analyze the influence of the stress limit values $\mathrm{K}_{I}, \mathrm{~K}_{I I}$ on the SDS in the vicinity of the plane domain boundary cutout vertex.

\section{References}

1. V.A. Kondratyev, Proceedings of the Moscow Mathematical Society, 16, 209-292 (1967)

2. O.K. Aksentyan, Applied Mathematics and Mechanics, 31, 1, 178-186 (1967)

3. K.S. Chobanyan, S.Kh. Gevorkyan, Proceedings of National Academy of Sciences of Armenia. Mechanics, Issue XXIV, 5, 16-24

4. V.Z. Parton, P.I. Perlin, Methods of the mathematical theory of elasticity, 688 (Moscow, Nauka)

5. G.P. Cherepanov, Mechanics of brittle fracture, 640 (Moscow, Nauka, 1974)

6. M. L. Williams, J. Appl. Mech, 19, 4, 526 (1952)

7. G.S. Vardanyan, L.Yu. Frishter, International journal for computational civil and structural engineering, 3, 2, 75-81 (2007)

8. S.P. Timoshenko, J. Goodier, Theory of elasticity, 576 (Moscow, Nauka, 1975)

9. G.S. Vardanyan, M.L. Mozgaleva, V.N. Savostyanov, L.Yu. Frishter, Proceedings of Universities Civil Engineering, 10, 28-31 (2003)

10. V.D. Kuliev, Singular boundary-value problems, 719 (Moscow, Nauka, 2005)

11. I.T. Denisyuk, Proceedings of Universities, Mathematics, 6, 70-74 (2000)

12. G.S. Vardanyan, V.N. Savostyanov, L.Yu. Frishter, Development of methods of experimental mechanics Moscow, IMASH RAS, 60-68 (2003)

13. L. Frishter, MATEC Web of Conferences, 117 (2017)

14. I.A. Razumovskiy, Interference-optical methods of deformable solid mechanics 240, (Moscow, MGTU named after N.E. Bauman, 2007)

15. Photoelasticity method. 3, 311 (Mpscow, Stroyizdat, 1975)

16. G.N. Albaut, M.V. Tabanyukhova, N.V. Kharinova Press Determination of the first stress intensity factor in elements with angle cutout. (Experimental Mechanics and Calculation of Structures, Kostinsky Readings), 166-175 (Moscow, Moscow State University, 2004) 